\title{
EXPRESSION OF EUKARYOTEIC INITIATION FACTOR 4E IN THE CHILDREN WITH MEDULLOBLASTOMA
}

\author{
Z. Salehi, F. Mashayekhi \\ Department of Cell and Molecular Biology, Islamic Azad University- Tonekabon Branch, Tonekabon, Iran
}

\begin{abstract}
Medulloblastoma, a neuroectodermal tumor arising in the cerebellum, is the most common malignant brain tumor in children, accounting for $10-20 \%$ of primary CNS neoplasms and approximately $40 \%$ of all posterior fossa tumors. Medulloblastoma is heterogeneous, being characterized by molecular subgroups that demonstrate distinct gene expression profiles. The cellular and molecular events involved in the progression of medulloblastoma are largely unknown. Increased protein synthesis is necessary for the transition of cells from quiescence to proliferation. Translational control is critical for the proper regulation of the cell cycle, tissue induction and growth. Eukaryotic initiation factor 4E (eIF4E), an important regulator of translation, plays critical roles in neo-plastic transformation and tumor progression. The aim of this study is to determine the pattern of eIF4E expression in the children with medulloblastoma.Tumor and normal adjacent tissue samples (as controls) were obtained from 28 children with medulloblastoma. eIF4E expression were determined by using Western blot analysis. In the medulloblastoma tissues, eIF4E level was elevated by a mean of $8.22 \pm 1.41$-fold when compared to normal adjacent tissues. eIF4E expression was higher in tumors compared to control tissues. The results from this study support the hypothesis that the overexpression of eIF4E can be involved in tumorigenesis and a possible role of eIF4E as a prognostic factor in medulloblastoma.
\end{abstract}

